\title{
Structural Dynamic Reanalysis of Cantilever Beam Using Polynomial Regression Method
}

\author{
C.Srikarrao ${ }^{1}$, K.Kumar $^{2}$, P.Pavan $\mathrm{Kumar}^{3}$, C. Mukul ${ }^{4}$,P.Vinay ${ }^{5}$ \\ ${ }^{1,3,4,5}$ (M.Tech-Student, Department of Mechanical Engineering, Jntuceh/Jntuh, Hyderabad, Telangana, India) \\ ${ }_{2}^{2}$ (B.Tech, Mechanical Engineering)
}

\begin{abstract}
Reanalysis methods are intended to analyze efficiently structures that are modified due to changes in the design. To find out the changes in the dynamic properties (natural frequency), solving of various simultaneous equations for Eigen values is necessary and is a length, breadth and depth. In this paper, the focus will be on reanalysis of a Cantilever beam. The natural frequency of the Cantilever beam will be calculated using Ansys, FEM (using MAT LAB software) and also using conventional equations of Cantilever Beam. These values are compared using polynomial regression method.
\end{abstract}

Keywords: frequency, mass matrix, physical parameters, stiffness matrix, regression method, ANSYS, Reanalysis

\section{Introduction}

Most optimal design procedures are iterative and require repeated analyses of structures obtained by progressive modifications in design variables. Reanalysis methods are intended to analyze efficiently structures that are modified due to changes in design. The object is to evaluate the structural modification within the frame of vibration analysis technology refers to technique to modify physical properties of a structure in order to change or optimize its dynamic properties. To find out the changes in the dynamic properties (natural frequency), solving of various simultaneous equations for the Eigen values is necessary and is lengthy process. To avoid this, changes in physical parameters are considered such as length, breadth and depth.

\section{Reanalysis}

Reanalysis methods are intended to analyze efficiently new designs using information from previous ones. One of the many advantages of the substructure technique is the possibility of repeating the analysis for one or more of the substructures making use of the work done on the others. This represents a significant saving of time when modifications once are required. Modification is invariably required in iterative processes for optimum design never the less, in the case of large structures the expenses are still too high. Therefore, development of techniques which are themselves based on previous analysis, and which obtained the condensed matrices of the substructures under modification, with little extra calculation time, can be very useful. "General Reanalysis Techniques" are very useful in solving medium size problems and are totally essential in the design of large structures. Some steps in a dynamic condensation process are particularly characterized by their computational effort, as for instance:

1. Stiffness matrix factorization

2. Resolution of certain systems of linear equation

3. Resolution of an Eigen problem to obtain the normal vibration modes

Reanalysis methods are intended to analyze efficiently structures that are modified due to changes in the design. The object is to evaluate the structural response for such changes without solving the complete set of modified simultaneous equations. The solution procedures usually use the original response of the structure. Development of structural modification techniques which are themselves based on the previous analysis. The modified matrices of the beam element structures are obtained, with little extra calculation time, can be very easy and useful. The General structural modification techniques are very useful in solving medium size structural problems as well as for the design of large structures also. The main object is to evaluate the dynamic characteristics for such changes without solving the total (or) complete set of modified equations.

\section{Finite Element Method Approach}

Initially the total structure of Cantilever beam is divided into small elements using successive levels of divisions. In finite element analysis the structure is discredited into a set of elements joined together at some points called nodes or nodal points. In analysis more number of elements will give more incisiveness results. Assay of stiffness and mass matrix are performed for each element separately and then globalized into a single matrix for the total system. The dynamic analysis of beam structure is $[\mathrm{K}-\lambda \mathrm{M}][\mathrm{X}]=0$ (1) 
Where $\mathrm{k}, \mathrm{m}$ are the stiffness and mass matrix respectively. The dynamic behavior of a damped structure [4] which is assumed to linear and discretized for $\mathrm{n}$ degrees of freedom can be described by the equation of motion. $\mathrm{M}+\mathrm{C}+\mathrm{Kx}=\mathrm{f}-------------(2)$

Where $\mathrm{M}, \mathrm{C}=\alpha \mathrm{M}+\beta \mathrm{K}$, and $\mathrm{K}$ are mass, damping and stiffness matrices, , and $\mathrm{X}$ are acceleration, velocity, displacement vectors of the structural points and "f" is force vector. Undamped homogeneous equation $\mathrm{M}+\mathrm{Kx}=0$. Provides the Eigen value problem $(\mathrm{k}-\lambda \mathrm{m})=0$.

Solution of above equation fetches the matrices eigen values $\lambda$ and eigen vector $\Theta$.

$\lambda=\left(\begin{array}{ccc}w_{1}^{2} & \cdots & \cdots \\ \cdots & \cdots & \cdots \\ \cdots & \cdots & w_{n}^{2}\end{array}\right), \phi=\left[\phi_{1}, \phi_{2}, \ldots ., \phi_{n}\right]$

The eigen vector will satisfy the orthonormal conditions $\phi^{T} M \phi=I, \phi^{T} K \phi=\lambda, \phi^{T} C \phi=\alpha I=\beta \lambda=\xi$, if we use the transformation $X=\phi q$ in the equation of motion, and premultiplying by $\phi^{T}$ we get $\phi^{T} M \phi q+\phi^{T} C \phi q+\phi^{T} K \phi q=\phi^{T} f$

It is vital note, that the matrices $M=\phi^{T} M \phi, C=\phi^{T} C \phi, K=\phi^{T} K \phi$

are not usually diagonalised by the eigenvectors of the original structure [3] Given an initial geometry Ŷand assuming a change $\Delta \mathrm{Y}$ in the design variables, the modified design is given by $Y=Y+\Delta Y$

The geometric variables Y usually represent coordinates of joints, but other choice for thesevariables is sometimes preferred. The displacement analysis equations for the initial design are $K r=R$. where $K=$ stiffness matrix corresponding to the design $\hat{Y}, R=$ load vector whose elements are usually assumed to be independent of the design variables and $r=$ nodal displacements computed $\hat{Y}$ at . The stiffness matrix and mass matrix of a typical plane truss element are

$$
\begin{aligned}
K & =\frac{E A}{l^{3}}\left(\begin{array}{cccc}
12 & 6 l_{e} & -12 & 6 l_{e} \\
6 l_{e} & 4 l_{e}^{2} & -6 l_{e} & 2 l_{e}^{2} \\
-12 & 6 l_{e} & 12 & 6 l_{e} \\
-6 l_{e} & 2 l_{e}^{2} & -6 l_{e} & 4 l_{e}^{2}
\end{array}\right) \text { and } \\
M & =\frac{\rho A_{e} l_{e}}{420}\left(\begin{array}{cccc}
156 & 22 l_{e} & 54 & -13 l_{e} \\
22 l_{e} & 4 l_{e}^{2} & 13 l_{e} & -3 l_{e}^{2} \\
54 & 13 l_{e} & 156 & -22 l_{e} \\
-13 l_{e} & -3 l_{e}^{2} & -22 l_{e} & 4 l_{e}^{2}
\end{array}\right)
\end{aligned}
$$

Where ' $A$ ' is the cross sectional area of the cantilever beam, ' $l$ ' is the length of the cantilever beam, ' $\rho$ ' is the density of the cantilever beam material

\section{Regression Method}

As with correlation, regression is used to analyze the relation between two continuous (scale) variables. However, regression is better suited for studying functional dependencies between factors. The term functional dependencyimplies that $\mathrm{X}$ [partially] determines the level of Y. For example, there is a function dependency between age and Blood pressure since as one ages, blood pressure increases. In contrast, there is no functional dependency between arms Length and leg length since increasing the length of an arm will have no effect on leg length (or vice versa).In addition, regression is better suited than correlation for studying samples in which the investigator fixes thedistribution of X. For example, if I decide to select ten 30-year-olds, ten 40-year olds, and ten 50-year-olds to study the relation between age and blood pressure, I have fixed the distribution of the $\mathrm{X}$ variable in the sample. This would necessitate the use of regression and (in theory) prevent the use of 
correlation.The most elementary type of regression model is the simple linear regression model, which can be expressed by the following equation $Y=\alpha+\beta X+\varepsilon$

It is important to note that linearregression does not actually test whether the data sampled from the population follow a linear relationship. It assumes linearity and attempts to find the best-fit straight line relationship based on the data sample. The dashed line shown in the figure (1) is the deterministic component, whereas the points represent the effect of random error.

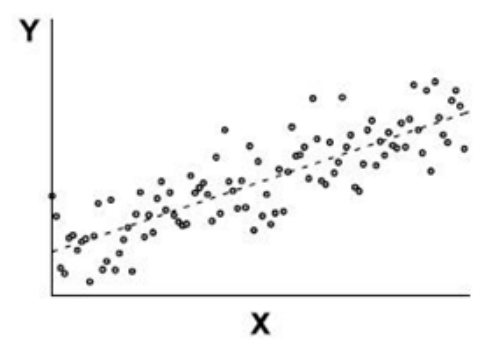

Figure 1: A linear model that incorporates a stochastic (random error) component.

\section{Assumptions of Standard Regression Analyses}

1. The subjects are randomly selected from a larger population. The same caveats apply here as with correlation analyses. The observations are independent. The variability of values around the line is Gaussian.

2. $\mathrm{X}$ and $\mathrm{Y}$ are not interchangeable. Regression models used in the vast majority of cases attempt to predict the dependent variable, $Y$, from the independent variable, $X$ and assume that the error in $X$ is negligible. In special cases where this is not the case, extensions of the standard regression techniques have been developed to account for non negligible error in X.

3. The relationship between $\mathrm{X}$ and $\mathrm{Y}$ is of the correct form, i.e., the expectation function (linear or nonlinear model) is appropriate to the data being fitted.

4. There are enough data points to provide a good sampling of the random error associated with the Experimental observations. In general, the minimum number of independent points can be no less than the number of parameters being estimated, and should ideally be significantly higher

5. The relationship is linear

6. The errors have the same variance

7. The errors are independent of each other

8. The errors are normally distributed

\section{Numerical Examples}

The polynomial regression method is applied to a simple cantilever beam structures. In finite element method, Discretization of the domain is the process of sub-dividing the domain (or structure) into many number of smaller parts. During discretization, the shapes, sizes, number and configuration of the elements have to be chosen carefully such that the original body is simulated as closely as possible without increasing the computational effort needed for the solution. The element must be made small enough to view and give usable results and to be large enough to reduce computational efforts. Small elements are generally desirable where the results are changing rapidly such as where the changes in geometry occur. Large elements can be used where the results are relatively constant. The discretized body or mesh is often created with mesh generation program or preprocessor programs available to the user. Figure (2) shows an example of creating a finite element for a cantilever beam.

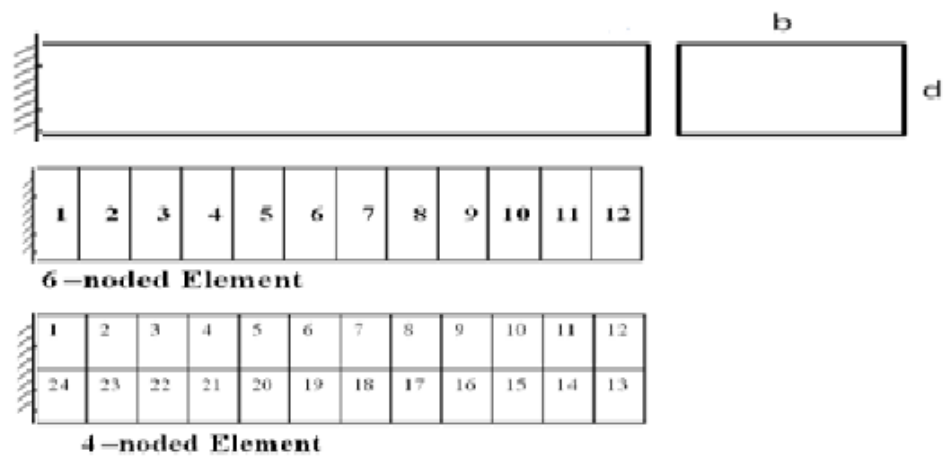

Figure 2: Discretized Element 
The polynomial equation for regression method,

$$
f_{n}=C_{1}+C_{2} B+C_{3} H+C_{4} B^{2}+C_{5} H^{2}+C_{6} B H
$$

These 3 values for the case study

\begin{tabular}{|l|l|}
\hline Young's modulus $(\mathrm{E})$ & $205 \times 10^{9} \mathrm{~N} / \mathrm{m}^{2}$ \\
\hline Density $(\rho)$ & $7850 \mathrm{Kg} / \mathrm{m}^{3}$ \\
\hline Cross section of area $(\mathrm{A})$ & $0.0756 \mathrm{~m}^{2}$ \\
\hline
\end{tabular}

Dynamic Reanalysis Of Cantilever Beam Using Conventional Equations:

9.1 Free Vibration Analysis of Beams using Conventional Equations:

he conventional equations for cantilever beam based on the mode shapes are given as follows:

(a)

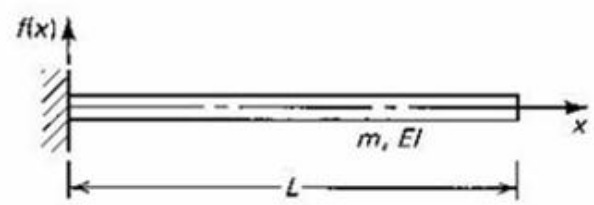

(b)

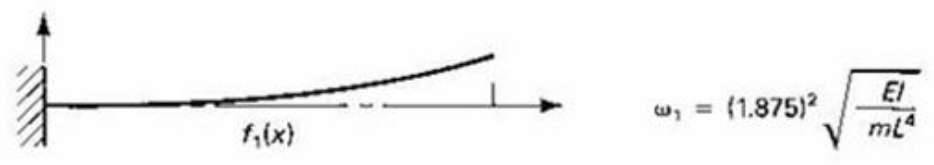

(c)

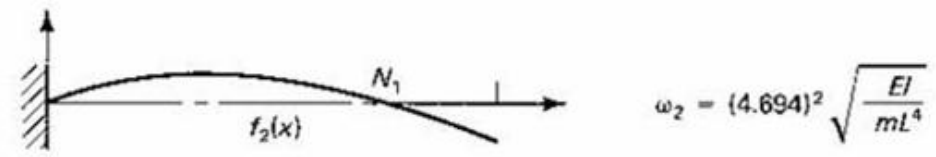

(d)

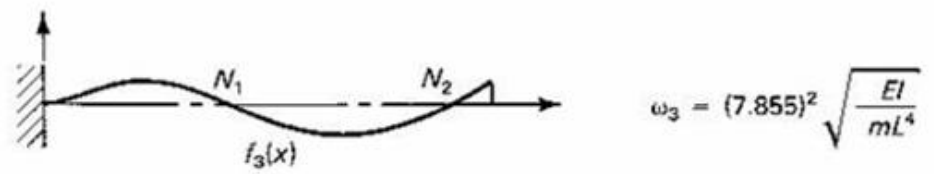

The first three undamped natural frequencies and mode shapes of cantilever beam

Case study :

A cantilever beam of $1.93 \mathrm{~m}$ length, $0.252 \mathrm{~m}$ breadth(b) and $0.3 \mathrm{~m}$ depth(d) is shown in above figure(3.2). The first mode of natural frequency values are calculated using conventional equation by considering the following situations:

1. Increasing the depth(d) of the beam by $5 \%$

2. Increasing the breadth(b) and depth(d) of the beam by $5 \%$

3. Decreasing the depth(d) of the beam by $5 \%$

4. Decreasing the breadth(b) and depth(d) by $5 \%$

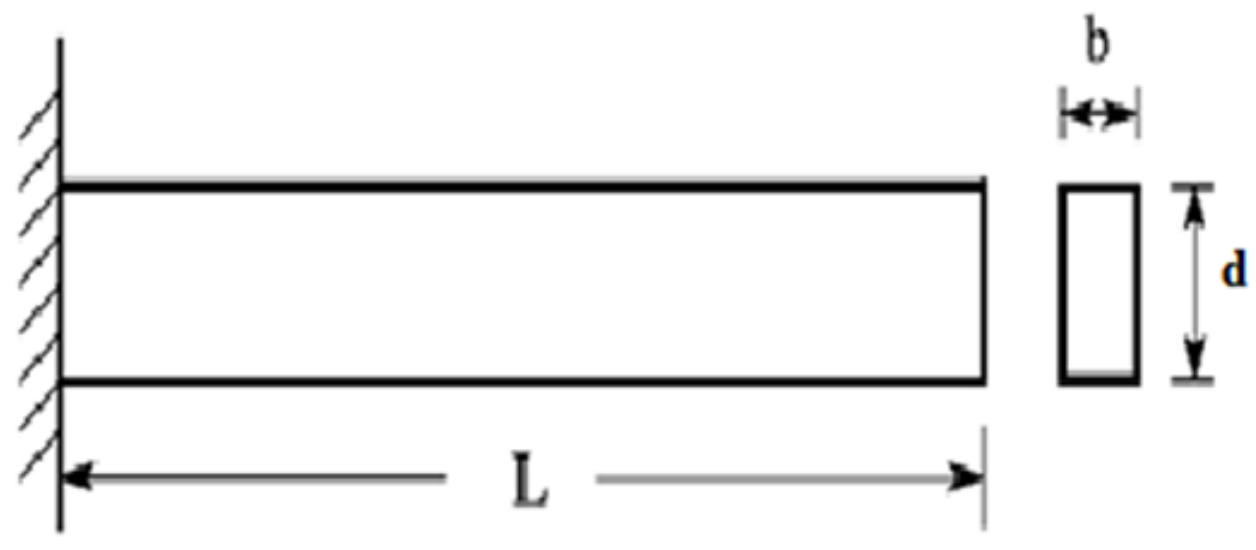

Fig. Cantilever Beam 
conventional results from first mode natural frequency formula:

The first mode of natural frequencies are obtained using conventional equation and the reanalysis of the beam is done by using polynomial regression method and the percentage errors are calculated.

$\omega_{n}=(1.875)^{2} \sqrt{\frac{E I}{m L^{4}}}$

For our paper $\mathbf{L}=\mathbf{1 . 9 3 m}, \mathrm{b}=\mathbf{0 . 2 5 2} \mathrm{m}, \mathrm{d}=\mathbf{0 . 3 \mathrm { m }}$

Therefore ,

Young's Modulu's, $E=205 \times 10^{9} \mathrm{~N} / \mathrm{m}^{2}$

Moment of inertia, $I=\frac{b d^{3}}{12} \quad m^{4}=0.000567$

Area, $\mathrm{A}=0.0756$

Density, $\rho=7850 \mathrm{Kg} / \mathrm{m}^{3}$

Hence,

$$
\omega_{n}=(1.875)^{2} \sqrt{\frac{\left[\left(205 \times 10^{9}\right)(0.000567)\right]}{\left[(7850)(0.0756)(1.93)^{4}\right]}}=417.6963305 \mathrm{rad} / \mathrm{sec}
$$

$=66.478 \mathrm{~Hz}$

Increasing the depth(d) of beam by $5 \%$

By using the polynomial regression method the natural frequencies of cantilever beam for increasing the depth(d) by $5 \%$ are as follows:

$f n=\alpha_{1}+\alpha_{2} b+\alpha_{3} d+\alpha_{4} b^{2}+\alpha_{5} d^{2}+\alpha_{6} b d$

$\alpha_{1}=-9.4658492835519595 \mathrm{E}-02$

$\alpha_{3}=2.0884918091567624 \mathrm{E}+02$

$$
\begin{gathered}
\alpha_{2}=-2.3853940192537948 \mathrm{E}-02 \\
\alpha_{4}=-6.0111929279216270 \mathrm{E}-03 \\
\alpha_{6}=5.2629993590750750 \mathrm{E}+01
\end{gathered}
$$

Table 3.3.1: Increasing the depth of beam by $5 \%$

\begin{tabular}{|c|c|c|c|l|}
\hline Breadth(b) & Depth(d) & $\boldsymbol{f n}$ (Conventional) & $\boldsymbol{f n}$ (Regression) & \% Error \\
\hline 0.252 & 0.3 & 66.47 & 66.47354 & -0.05806 \\
\hline 0.252 & 0.315 & 69.80235751 & 69.79917 & -0.05523 \\
\hline 0.252 & 0.33 & 73.12627928 & 73.12451 & -0.05311 \\
\hline 0.252 & 0.345 & 76.45020107 & 76.44955 & -0.05154 \\
\hline 0.252 & 0.36 & 79.77412285 & 79.7743 & -0.05047 \\
\hline 0.252 & 0.375 & 83.09804464 & 83.09875 & -0.04984 \\
\hline 0.252 & 0.39 & 86.42196642 & 86.42291 & -0.0496 \\
\hline 0.252 & 0.405 & 89.74588821 & 89.74677 & -0.04971 \\
\hline 0.252 & 0.42 & 93.06980999 & 93.07034 & -0.05013 \\
\hline 0.252 & 0.435 & 96.39373178 & 96.39361 & -0.05082 \\
\hline 0.252 & 0.45 & 99.71765357 & 99.71659 & -0.05176 \\
\hline
\end{tabular}

Increasing the breadth(b) and $\operatorname{depth}(d)$ of the beam by $5 \%$

By using the polynomial regression method the natural frequencies of cantilever beam for increasing the breadth(b) and depth(d) by $5 \%$ are as follows:

$f n=\alpha_{1}+\alpha_{2} b+\alpha_{3} d+\alpha_{4} b^{2}+\alpha_{5} d^{2}+\alpha_{6} b d$

$\alpha_{1}=1.6257664335197344 \mathrm{E}-02 \quad \alpha_{2}=1.0908943441650720 \mathrm{E}+02$

$\alpha_{3}=1.2986837430536571 \mathrm{E}+02 \quad \alpha_{4}=3.9719636556959870 \mathrm{E}-02$

$\alpha_{5}=5.6292001923083035 \mathrm{E}-02 \alpha_{6}=4.7285281615472741 \mathrm{E}-02$

Increasing the breadth(b) and $\operatorname{depth}(d)$ by $5 \%$

\begin{tabular}{|c|l|c|c|c|}
\hline Breadth(b) & Depth(d) & $\boldsymbol{f n}$ (Conventional) & fn (Regression) & \%Error \\
\hline 0.252 & 0.3 & 66.51215 & 66.47847 & -0.05064 \\
\hline 0.2646 & 0.315 & 69.83776 & 69.80217 & -0.05097 \\
\hline 0.2772 & 0.33 & 73.16337 & 73.12592 & -0.05119 \\
\hline 0.2898 & 0.345 & 76.48898 & 76.44973 & -0.05131 \\
\hline 0.3024 & 0.36 & 79.81459 & 79.77359 & -0.05136 \\
\hline 0.315 & 0.375 & 83.14019 & 83.09751 & -0.05134 \\
\hline
\end{tabular}


Structural Dynamic Reanalysis of Cantilever Beam Using Polynomial Regression Method

\begin{tabular}{|l|l|l|l|l|}
\hline 0.3276 & 0.39 & 86.4658 & 86.42149 & -0.05125 \\
\hline 0.3402 & 0.405 & 89.79141 & 89.74552 & -0.05111 \\
\hline 0.3528 & 0.42 & 93.11702 & 93.06961 & -0.05091 \\
\hline 0.3654 & 0.435 & 96.44262 & 96.39375 & -0.05068 \\
\hline 0.378 & 0.45 & 99.76823 & 99.71795 & -0.0504 \\
\hline
\end{tabular}

Decreasing the depth(d) of the beam by $5 \%$

By using the polynomial regression method the natural frequencies of cantilever beam for decreasing the depth(d) by $5 \%$ are as follows:

$f n=\alpha_{1}+\alpha_{2} b+\alpha_{3} d+\alpha_{4} b^{2}+\alpha_{5} d^{2}+\alpha_{6} b d$

$\alpha_{1}=-3.2325620284851558 \mathrm{E}-02$

$\alpha_{3}=2.0868197121130873 \mathrm{E}+02$

$\alpha_{2}=-8.1460563085897775 \mathrm{E}-03$

$\alpha_{5}=-5.4208754208936583 \mathrm{E}-01 \alpha_{6}=5.2587856745250306 \mathrm{E}+01$

Decreasing the depth(d) of the beam by $5 \%$

\begin{tabular}{|c|c|c|c|c|}
\hline Breadth(b) & Depth(d) & $\boldsymbol{f n}$ (Conventional) & $\boldsymbol{f n}$ (Regression) & \% Error \\
\hline 0.252 & 0.3 & 66.47 & 66.47354 & -0.05806 \\
\hline 0.252 & 0.285 & 63.18655 & 63.17268 & -0.02194 \\
\hline 0.252 & 0.27 & 59.86094 & 59.84818 & -0.02131 \\
\hline 0.252 & 0.255 & 56.53533 & 56.52344 & -0.02103 \\
\hline 0.252 & 0.24 & 53.20972 & 53.19845 & -0.02118 \\
\hline 0.252 & 0.225 & 49.88412 & 49.87322 & -0.02184 \\
\hline 0.252 & 0.21 & 46.55851 & 46.54775 & -0.02311 \\
\hline 0.252 & 0.195 & 43.2329 & 43.22203 & -0.02514 \\
\hline 0.252 & 0.18 & 39.90729 & 39.89607 & -0.02813 \\
\hline 0.252 & 0.165 & 36.58168 & 36.56986 & -0.03232 \\
\hline 0.252 & 0.15 & 33.25608 & 33.24341 & -0.03809 \\
\hline
\end{tabular}

Decreasing the Breadth(b) and Depth(d) of the beam by $5 \%$

By using the polynomial regression method the natural frequencies of cantilever beam for decreasing the breadth(b) and depth(d) by $5 \%$ are as follows:

$f n=\alpha_{1}+\alpha_{2} b+\alpha_{3} d+\alpha_{4} b^{2}+\alpha_{5} d^{2}+\alpha_{6} b d$

$\alpha_{1}=-4.0920431654701064 \mathrm{E}+00$

$\alpha_{3}=4.8636570319929723 \mathrm{E}+02$

$\alpha_{2}=-2.7795014096424597 \mathrm{E}+02$

$\alpha_{4}=-3.4603540586310110 \mathrm{E}+03$

$\alpha_{5}=-3.4474950483908215 \mathrm{E}+03 \alpha_{6}=6.9409522011659174 \mathrm{E}+03$

Decreasing the Breadth(b) and $\operatorname{Depth}(d)$ of the beam by $5 \%$

\begin{tabular}{|c|c|c|c|l|}
\hline Breadth(b) & Depth(d) & $\boldsymbol{f n}$ (Conventional) & $\boldsymbol{f n}$ (Regression) & \%Error \\
\hline 0.252 & 0.3 & 66.51215 & 66.48934 & -0.0343 \\
\hline 0.2394 & 0.285 & 63.18655 & 63.2113 & 0.03918 \\
\hline 0.2268 & 0.27 & 59.86094 & 59.90684 & 0.076683 \\
\hline 0.2142 & 0.255 & 56.53533 & 56.57596 & 0.071858 \\
\hline 0.2016 & 0.24 & 53.20972 & 53.21865 & 0.016769 \\
\hline 0.189 & 0.225 & 49.88412 & 49.83491 & -0.09864 \\
\hline 0.1674 & 0.21 & 46.55851 & 46.51493 & -0.0936 \\
\hline 0.1548 & 0.195 & 43.2329 & 43.23057 & -0.00539 \\
\hline 0.1422 & 0.18 & 39.90729 & 39.91978 & 0.031292 \\
\hline 0.1296 & 0.165 & 36.58168 & 36.58257 & 0.002415 \\
\hline 0.117 & 0.15 & 33.25608 & 33.21893 & -0.1117 \\
\hline
\end{tabular}

Dynamic Analysis Of Cantilever Beam Using Ansys

Free Vibration Analysis of Beams using ANSYS:

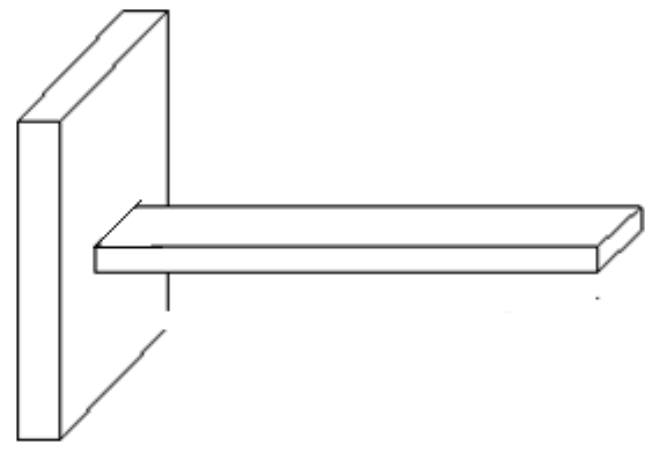




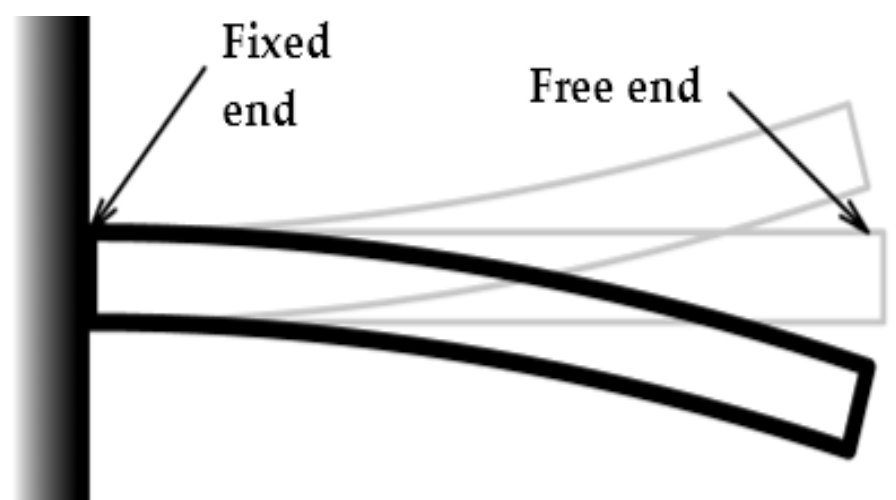

Beam under free Vibration

\section{Modal Analysis:}

Any physical system can vibrate. The frequencies at which vibration naturally occurs, and the modal shapes which the vibrating system assumes are properties of the system, and can be determined analytically using Modal Analysis.Analysis of vibration modes is a critical component of a design, but is often overlooked. Structural elements such as complex steel floor systems can be particularly prone to perceptible vibration, irritating building occupants or disturbing sensitive equipment. Inherent vibration modes in structural components or mechanical support systems can shorten equipment life, and cause premature or completely unanticipated failure, oftenresulting in hazardous situations. Detailed fatigue analysis is often required to assess the potential for failure or damage resulting from the rapid stress cycles of vibration.

Detailed seismic qualification also requires an understanding of the natural vibration modes of a system, as the large amount of energy acting on a system during seismic activity varies with frequency.The goal of modal analysis in structural mechanics is to determine the natural mode shapes and frequencies of an object or structure during free vibration. It is common to use the finite element method (FEM) to perform this analysis because, like other calculations using the FEM, the object being analyzed can have arbitrary shape and the results of the calculations are acceptable. The types of equations which arise from modal analysis are those seen in eigensystems. The physical interpretation of theeigenvalues and eigenvectors which come from solving the system are that they represent the frequencies and corresponding mode shapes. Sometimes, the only desired modes are the lowest frequencies because they can be the most prominent modes at which the object will vibrate, dominating all the higher frequency modes. It is also possible to test a physical object to determine its natural frequencies and mode shapes. This is called an Experimental Modal Analysis. The results of the physical test can be used to calibrate a finite element model to determine if the underlying assumptions made were correct (for example, correct material properties and boundary conditions were used).

\section{Procedure for modal in ANSYS}

1. Build the model of cantilever beam as shown in figure 4.1

2. Define the material properties such as young's modulus and density etc.,

3. Apply boundary conditions

4. Enter the ANSYS solution processor in which analysis type is taken as modal analysis, and by taking mode extraction method, by defining number of modes to be extracted.

5. Solve the problem using current LS command from the tool bar.

Here eigen values analysis of cantilever beam was carried out.

\section{Physical Properties:}

The physical properties of the beam are taken as follows:

\section{Physical Properties}

\begin{tabular}{|c|l|}
\hline Young's modulus(E) & $205 \times 10^{9} \mathrm{~N} / \mathrm{m}^{2}$ \\
\hline Density( $(\rho)$ & $7850 \mathrm{Kg} / \mathrm{m}^{3}$ \\
\hline Length(l) & $1.93 \mathrm{~m}$ \\
\hline Breadth(b) & $0.252 \mathrm{~m}$ \\
\hline Depth(d) & $0.3 \mathrm{~m}$ \\
\hline
\end{tabular}

\section{Case study:}

A cantilever beam of length $1.93 \mathrm{~m}$, breadth(b) of $0.252 \mathrm{~m}$ and depth(d) of $0.3 \mathrm{~m}$ shown in figure(4.1) is divided into 12 elements equally. Natural frequencies of the cantilever beam are calculated by considering the following situations: 
1. Increasing the depth(d) of the beam by $5 \%$

2. Increasing the breadth(b) and depth(d) of the beam by $5 \%$

3. Decreasing the depth(d) of the beam by $5 \%$

4. Decreasing the breadth(b) and depth(d) of the beam by $5 \%$

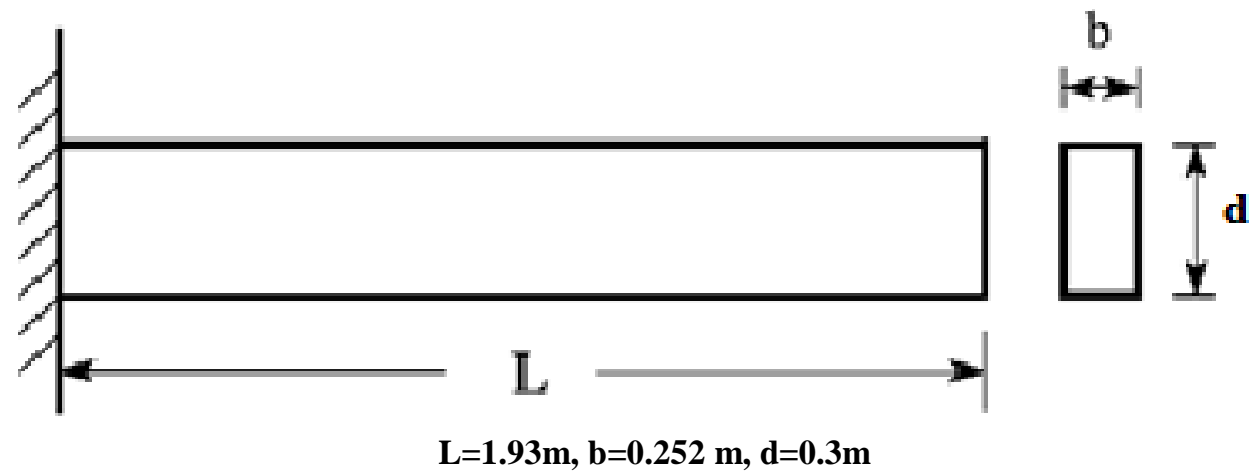

Figure : Cantilever beam

Results from ANSYS for case study:

The Modal analysis of cantilever beam has been carried out by using ANSYS10.0

Increasing the depth(d) of the beam by $5 \%$

By using ANSYS software the results for natural frequency for increasing the depth of the cantilever beam by $5 \%$ are calculated.

$f n=\alpha_{1}+\alpha_{2} \mathrm{~b}+\alpha_{3} \mathrm{~d}+\alpha_{4} \mathrm{~b}^{2}+\alpha_{5} \mathrm{~d}^{2}+\alpha_{6} \mathrm{bd}$

$\alpha_{1}=-5.0991185667651062 \mathrm{E}-01 \alpha_{2}=-1.2849778788034882 \mathrm{E}-01$

$\alpha_{3}=2.1262898807318032 \mathrm{E}+02 \alpha_{4}=-3.2381442545194261 \mathrm{E}-02$

$\alpha_{5}=-1.2442372442372843 \mathrm{E}+01 \alpha_{6}=5.3582504994441798 \mathrm{E}+01$

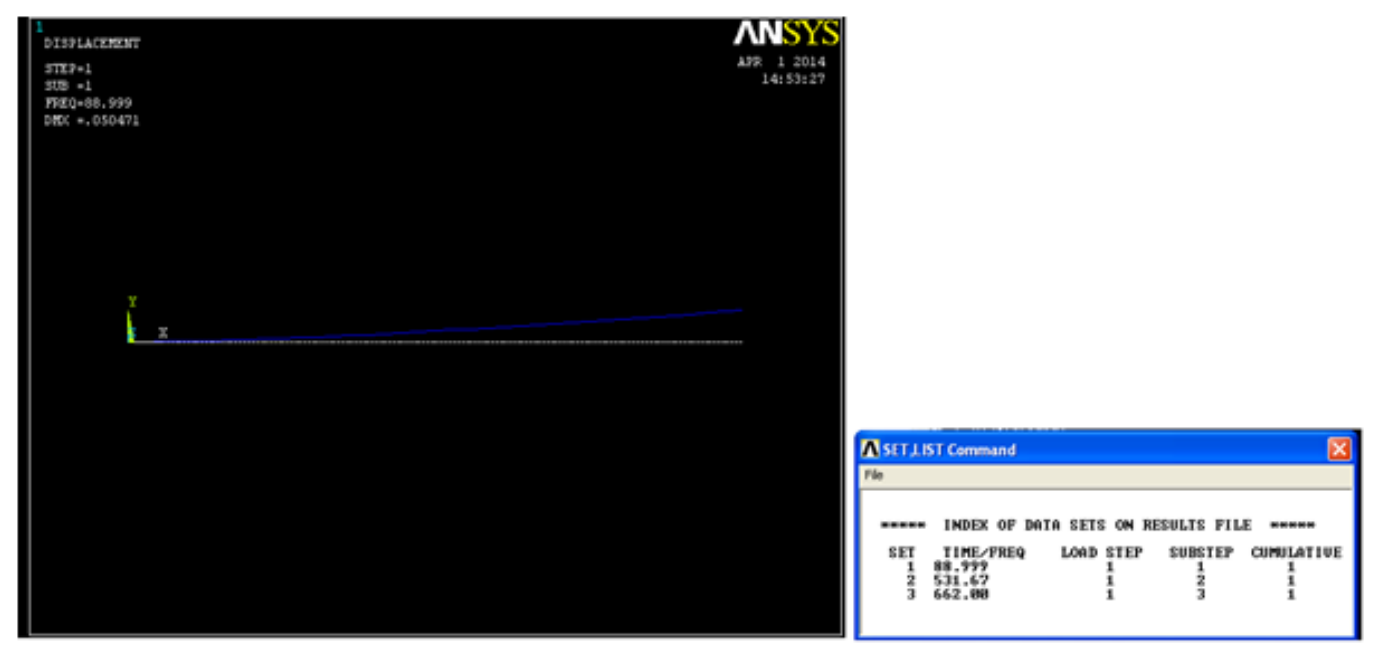

ANSYS answer for table 4.4.1(under lined in table)

Increasing the depth(d) of the beam by $5 \%$

\begin{tabular}{|c|l|r|r|c|}
\hline Breadth(b) & Depth(d) & $\boldsymbol{f n}$ (ANSYS) & $\boldsymbol{f n}$ (Regression) & \%Error \\
\hline 0.252 & 0.3 & 66.177 & 66.17537 & -0.00246 \\
\hline 0.252 & 0.315 & 69.452 & 69.45257 & 0.000816 \\
\hline 0.252 & 0.33 & 72.723 & 72.72416 & 0.001599 \\
\hline 0.252 & 0.345 & 75.989 & 75.99016 & 0.001528 \\
\hline 0.252 & 0.36 & 79.25 & 79.25056 & 0.000706 \\
\hline 0.252 & 0.375 & 82.506 & 82.50536 & -0.00078 \\
\hline 0.252 & 0.39 & 85.755 & 85.75456 & -0.00051 \\
\hline$\underline{0.252}$ & $\underline{0.405}$ & $\underline{88.999}$ & $\underline{88.99816}$ & $\underline{-0.00094}$ \\
\hline 0.252 & 0.42 & 92.237 & 92.23616 & -0.00091 \\
\hline 0.252 & 0.435 & 95.469 & 95.46857 & -0.00045 \\
\hline 0.252 & 0.45 & 98.694 & 98.69537 & 0.001389 \\
\hline
\end{tabular}


Increasing the breadth(b) and $\operatorname{depth}(\mathrm{d})$ of the beam by $5 \%$

From the ANSYS the results of natural frequency for increasing the breadth and depth of cantilever beam by $5 \%$ are calculated.

$f n=\alpha_{1}+\alpha_{2} b+\alpha_{3} d+\alpha_{4} b^{2}+\alpha_{5} d^{2}+\alpha_{6} b d$

$\alpha_{1}=-1.1349090909091647 \mathrm{E}+01$

$\alpha_{2}=1.4024644664279012 \mathrm{E}+02$

$\alpha_{3}=1.6696005552713106 \mathrm{E}+02 \alpha_{4}=-2.8701379021631737 \mathrm{E}+01$

$\alpha_{5}=-4.0676557570339845 \mathrm{E}+01 \alpha_{6}=-3.4168308359085330 \mathrm{E}+01$
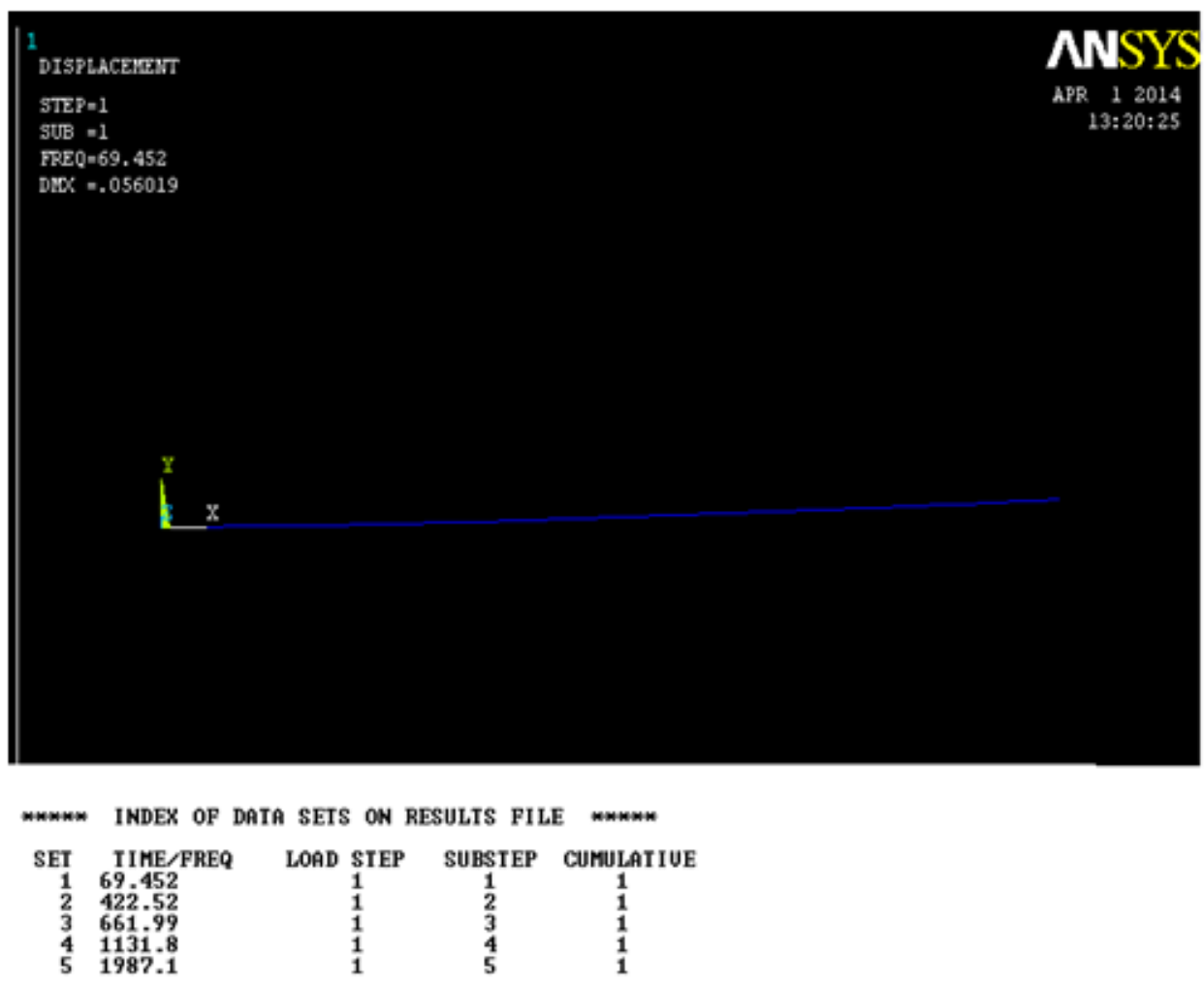

\section{ANSYS answer for}

Table 4.4.2(under lined in table)

Increasing the breadth(b) and $\operatorname{depth}(d)$ of the beam by $5 \%$

\begin{tabular}{|c|l|c|c|c|}
\hline Breadth(b) & Depth(d) & $\boldsymbol{f n ( A N S Y S )}$ & $\boldsymbol{f n}$ (Regression) & \%Error \\
\hline 0.252 & 0.3 & 66.177 & 66.01436 & -0.24576 \\
\hline 0.2646 & 0.315 & $\underline{69.452}$ & $\underline{69.45904}$ & 0.010131 \\
\hline 0.2772 & 0.33 & 72.723 & 72.86338 & 0.193028 \\
\hline 0.2898 & 0.345 & 76.149 & 76.22738 & 0.102932 \\
\hline 0.3024 & 0.36 & 79.25 & 79.55105 & 0.37988 \\
\hline 0.315 & 0.375 & 82.506 & 82.83439 & 0.398024 \\
\hline 0.3276 & 0.39 & 87.304 & 86.0774 & -1.40498 \\
\hline 0.3402 & 0.405 & 88.999 & 89.28007 & 0.315816 \\
\hline 0.3528 & 0.42 & 92.237 & 92.44241 & 0.2227 \\
\hline 0.3654 & 0.435 & 95.469 & 95.56442 & 0.099947 \\
\hline 0.378 & 0.45 & 98.694 & 98.64609 & -0.04854 \\
\hline
\end{tabular}

Decreasing the $\operatorname{depth}(\mathrm{d})$ of the beam by5\%

From ANSYS the results of natural frequency for decreasing the depth of the cantilever beam by $5 \%$ are calculated.

$f n=\alpha_{1}+\alpha_{2} b+\alpha_{3} d+\alpha_{4} b^{2}+\alpha_{5} d^{2}+\alpha_{6} b d$

$\alpha_{1}=-1.1399224458860556 \mathrm{E}-01$

$\alpha_{2}=-2.8726045128877331 \mathrm{E}-02$

$\alpha_{3}=2.0995566247864440 \mathrm{E}+02 \alpha_{4}=-7.2389633729237346 \mathrm{E}-03$

$\alpha_{5}=-7.6094276094307034 \mathrm{E}+00 \alpha_{6}=5.2908826944618909 \mathrm{E}+01$ 


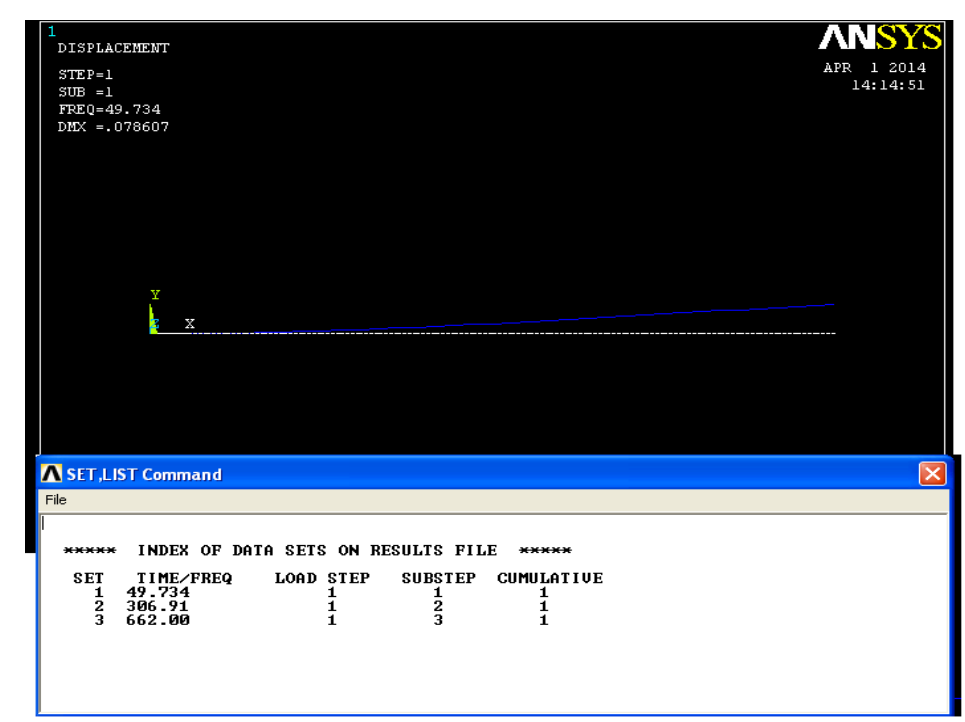

ANSYS answer for table 4.4.3(under lined in table)

Table : Decreasing the depth of the beam by $5 \%$

\begin{tabular}{|r|r|r|r|r|}
\hline Breadth(b) & Depth(d) & $\boldsymbol{f n}$ (ANSYS) & $\boldsymbol{f n}$ (Regression) & \%Error \\
\hline 0.252 & 0.3 & 66.177 & 66.177 & $-3.10606 \mathrm{E}-06$ \\
\hline 0.252 & 0.285 & 62.896 & 62.89751 & 0.002399 \\
\hline 0.252 & 0.27 & 59.612 & 59.61153 & -0.00079 \\
\hline 0.252 & 0.255 & 56.323 & 56.32212 & -0.00156 \\
\hline 0.252 & 0.24 & 53.03 & 53.02929 & -0.00134 \\
\hline$\underline{0.252}$ & $\underline{0.225}$ & $\underline{49.734}$ & $\underline{49.73304}$ & $\underline{-0.00194}$ \\
\hline 0.252 & 0.21 & 46.434 & 46.43336 & -0.00138 \\
\hline 0.252 & 0.195 & 43.131 & 43.13025 & -0.00173 \\
\hline 0.252 & 0.18 & 39.82 & 39.82373 & 0.00936 \\
\hline 0.252 & 0.165 & 36.511 & 36.51378 & 0.007603 \\
\hline 0.252 & 0.15 & 33.2004 & 33.2004 & -0.01084 \\
\hline
\end{tabular}

Decreasing the breadth(b) and depth(d) of the beam by $5 \%$

From ANSYS the results of natural frequency for decreasing the breadth and depth of the cantilever beam by $5 \%$ are calculated.

$f n=\alpha_{1}+\alpha_{2} b+\alpha_{3} \mathrm{~d}+\alpha_{4} b^{2}+\alpha_{5} d^{2}+\alpha_{6} b d$

$\alpha_{1}=-2.1918705035985325 \mathrm{E}-01 \alpha_{2}=-5.6209432233832217 \mathrm{E}+00$

$\alpha_{3}=2.2882398353751557 \mathrm{E}+00 \alpha_{4}=3.9293012483360641 \mathrm{E}+02$

$\alpha_{5}=-5.5896385612114621 \mathrm{E}+00 \alpha_{6}=-3.7390586882739022 \mathrm{E}+01$

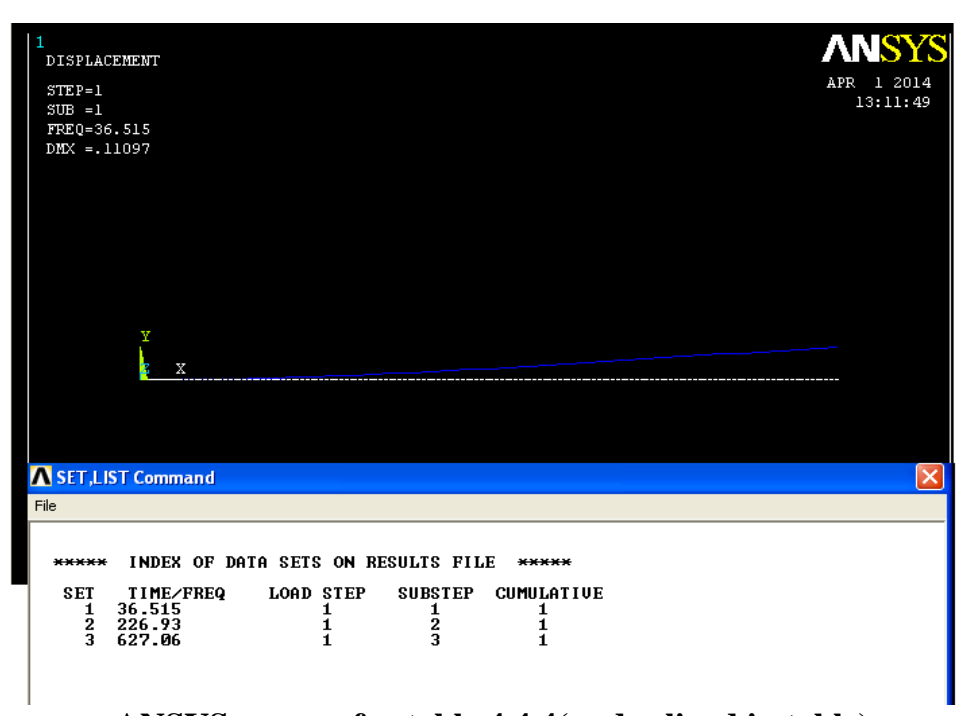

ANSYS answer for table 4.4.4(under lined in table) 
Table: Decreasing the breadth(b) and $\operatorname{depth}(d)$ of the beam by $5 \%$

\begin{tabular}{|c|c|c|c|l|}
\hline Breadth(b) & Depth(d) & $\boldsymbol{f n}$ (ANSYS) & $\boldsymbol{f n}$ (Regression) & \%Error \\
\hline 0.252 & 0.3 & 66.177 & 66.177 & $-3.10606 \mathrm{E}-06$ \\
\hline 0.2394 & 0.285 & 62.896 & 62.89683 & 0.001318021 \\
\hline 0.2268 & 0.27 & 59.612 & 59.61249 & 0.000817551 \\
\hline 0.2142 & 0.255 & 56.327 & 56.32397 & -0.005373847 \\
\hline 0.2016 & 0.24 & 53.03 & 53.03129 & 0.002425279 \\
\hline 0.189 & 0.225 & 49.734 & 49.73443 & 0.000857594 \\
\hline 0.1674 & 0.21 & 46.434 & 46.43307 & -0.002001758 \\
\hline 0.1548 & 0.195 & 43.131 & 43.13173 & 0.001691351 \\
\hline 0.1422 & 0.18 & 39.824 & 39.82622 & 0.00556405 \\
\hline 0.1296 & 0.165 & 36.515 & 36.51653 & 0.00418868 \\
\hline 0.117 & 0.15 & 33.204 & 33.20267 & -0.004004025 \\
\hline
\end{tabular}

\section{Dynamic Analysis Of Cantilever Beam Usingfinite Element Method Free vibration Analysis of the Beam using Finite Element Method:}

The polynomial regression method is applied to a simple beam structures. In finite element method, Discretization means dividing the body into an equivalent system of finite elements with associated nodes. The element must be made small enough to view and give usable results and to be large enough to reduce computational efforts. Small elements are generally desirable where the results are changing rapidly such as where the changes in geometry occur. Large elements can be used where the results are relatively constant. The discretized body or mesh is often created with mesh generation program or pre-processor programs available to the user. Figure shows an example of creating a finite element for a cantilever beam.

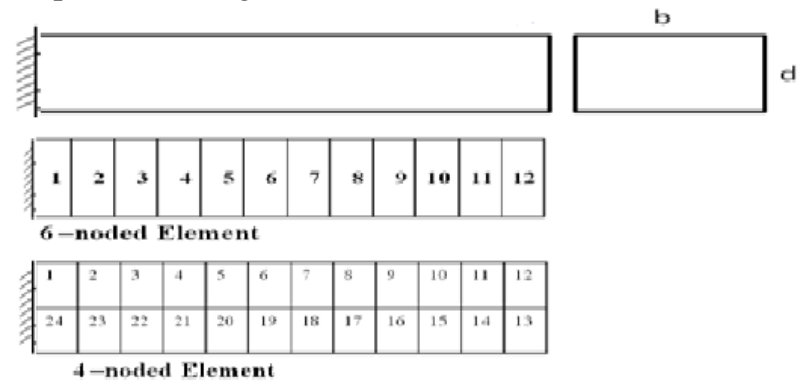

Discretized Element

The values of young's modulus(E), density( $\rho)$, length(l), breadth(b), depth(d) for the case study are follows:

Table: Element Properties

\begin{tabular}{|l|l|}
\hline Young's modulus(E) & $205 \times 10^{9}$ \\
\hline Density $(\rho)$ & $7850 \mathrm{Kg} / \mathrm{m}^{2}$ \\
\hline Length(l) & $1.93 \mathrm{~m}$ \\
\hline Breadth(b) & $0.252 \mathrm{~m}$ \\
\hline Depth(d) & $0.3 \mathrm{~m}$ \\
\hline
\end{tabular}

\section{Case study:}

A cantilever beam of length $1.93 \mathrm{~m}$, breadth(b) of $0.252 \mathrm{~m}$ and depth(d) of $0.3 \mathrm{~m}$ shown in figure(4.1) is divided into 12 elements equally. Natural frequencies of the cantilever beam are calculated by considering the following situations:

1. Increasing the depth(d) of the beam by $5 \%$

2. Increasing the breadth(b) and depth(d) of the beam by $5 \%$

3. Decreasing the depth(d) of the beam by $5 \%$

4. Decreasing the breadth(b) and depth(d) of the beam by $5 \%$

5 .

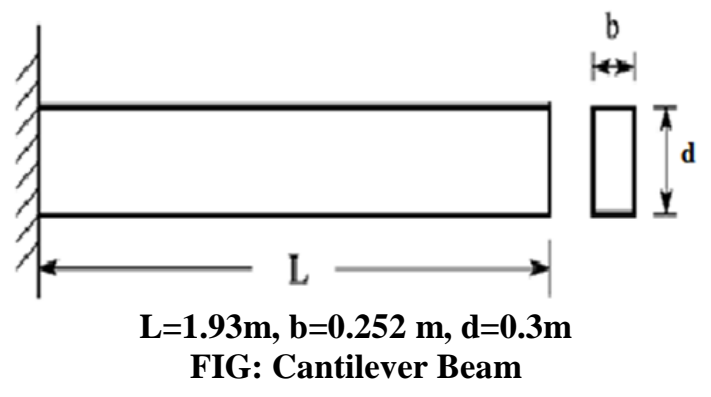


Increasing the depth of the beam by $5 \%$

By using the polynomial regression method the natural frequencies of cantilever beam for increasing the depth(d) by $5 \%$ are as follows:

$f n=\alpha_{1}+\alpha_{2} b+\alpha_{3} d+\alpha_{4} b^{2}+\alpha_{5} d^{2}+\alpha_{6} b d$

$\alpha_{1}=4.0431800437165233 \mathrm{E}+00$

$\alpha_{2}=1.0188813710176134 \mathrm{E}+00$

$\alpha_{3}=1.6334663458418589 \mathrm{E}+02$

$\alpha_{5}=3.7744208961411381 \mathrm{E}+01 \alpha_{6}=4.1163351915215031 \mathrm{E}+01$

Table: Increasing the depth of the beam by $5 \%$

\begin{tabular}{|c|c|c|c|c|}
\hline Breadth(b) & Depth(d) & $f \mathrm{n}$ (FEM) & $f$ n (Regression) & \%Error \\
\hline 0.252 & 0.3 & 59.9 & 59.82916 & -0.11826 \\
\hline 0.252 & 0.315 & 62.996 & 62.78315 & -0.33788 \\
\hline 0.252 & 0.33 & 65.99655 & 65.75412 & -0.36733 \\
\hline 0.252 & 0.345 & 67.54248 & 68.74208 & 1.776069 \\
\hline 0.252 & 0.36 & 71.99541 & 71.74702 & -0.345 \\
\hline 0.252 & 0.375 & 74.99559 & 74.76895 & -0.30221 \\
\hline 0.252 & 0.39 & 77.99567 & 77.80786 & -0.24079 \\
\hline 0.252 & 0.405 & 80.98938 & 80.86376 & -0.15512 \\
\hline 0.252 & 0.42 & 83.99401 & 83.93664 & -0.06831 \\
\hline 0.252 & 0.435 & 86.99386 & 87.0265 & 0.037519 \\
\hline 0.252 & 0.45 & 89.99364 & 90.13336 & 0.155249 \\
\hline
\end{tabular}

Increasing the breadth(b) and $\operatorname{depth}(d)$ by $5 \%$

By using the polynomial regression method the natural frequencies of cantilever beam for increasing the breadth(b) and depth(d) by $5 \%$ are as follows:

$f n=\alpha_{1}+\alpha_{2} b+\alpha_{3} d+\alpha_{4} b^{2}+\alpha_{5} d^{2}+\alpha_{6} b d$

$\alpha_{1}=1.0321982796935379 \mathrm{E}-02$

$$
\alpha_{2}=9.8463663462260911 \mathrm{E}+01
$$

$\alpha_{3}=\quad 1.1721864697888201 \mathrm{E}+02 \alpha_{4}=\quad 2.5061115923598720 \mathrm{E}-02 \alpha_{5}=$

$\alpha_{6}=2.9834661813843866 \mathrm{E}-02$

Table: Increasing the breadth(b) and $\operatorname{depth}(d)$ by $5 \%$

\begin{tabular}{|c|l|r|r|l|}
\hline Breadth(b) & Depth(d) & $f$ n (FEM) & $f$ n (Regression) & \%Error \\
\hline 0.252 & 0.3 & 59.99529 & 59.9958 & 0.000852 \\
\hline 0.2646 & 0.315 & 62.99601 & 62.99545 & -0.0009 \\
\hline 0.2772 & 0.33 & 65.99655 & 65.99513 & -0.00216 \\
\hline 0.2898 & 0.345 & 68.99509 & 68.99484 & -0.00036 \\
\hline 0.3024 & 0.36 & 71.99013 & 71.99459 & -0.006197 \\
\hline 0.315 & 0.375 & 74.99593 & 74.99437 & -0.00207 \\
\hline 0.3276 & 0.39 & 77.99567 & 77.99419 & -0.00189 \\
\hline 0.3402 & 0.405 & 80.99407 & 80.99405 & $-3.1 \mathrm{E}-05$ \\
\hline 0.3528 & 0.42 & 83.99401 & 83.99394 & $-8.6 \mathrm{E}-05$ \\
\hline 0.3654 & 0.435 & 86.99366 & 86.99387 & 0.00023 \\
\hline 0.378 & 0.45 & 89.99364 & 89.99383 & 0.000205 \\
\hline
\end{tabular}

Decreasing the depth(d) of the beam by $5 \%$

By using the polynomial regression method the natural frequencies of cantilever beam for decreasing the depth(d) by $5 \%$ are as follows:

$f n=\alpha_{1}+\alpha_{2} b+\alpha_{3} d+\alpha_{4} b^{2}+\alpha_{5} d^{2}+\alpha_{6} b d$

$\alpha_{1}=-3.2325620284851558 \mathrm{E}-02$

$\alpha_{3}=2.0868197121130873 \mathrm{E}+02$

$\alpha_{2}=-8.1460563085897775 \mathrm{E}-03$

$\alpha_{5}=-5.4208754208936583 \mathrm{E}-01 \alpha_{6}=5.2587856745250306 \mathrm{E}+01$

Table: Decreasing the depth(d) of the beam by $5 \%$

\begin{tabular}{|r|r|r|r|r|}
\hline Breadth(b) & Depth(d) & \multicolumn{1}{|l|}{$\boldsymbol{f n}$ (FEM) } & fn (Regression) & \%Error \\
\hline 0.252 & 0.3 & 59.99529 & 59.99549 & 0.00033 \\
\hline 0.252 & 0.285 & 56.99658 & 56.99608 & -0.00088 \\
\hline 0.252 & 0.27 & 53.99553 & 53.99634 & 0.0015 \\
\hline 0.252 & 0.255 & 50.99668 & 50.99658 & -0.00019 \\
\hline 0.252 & 0.24 & 47.99703 & 47.99682 & -0.00043 \\
\hline 0.252 & 0.225 & 44.99724 & 44.99704 & -0.00046 \\
\hline 0.252 & 0.21 & 41.99731 & 41.99724 & -0.00015 \\
\hline 0.252 & 0.195 & 38.99751 & 38.99744 & -0.00017 \\
\hline 0.252 & 0.18 & 35.99753 & 35.99762 & 0.000267 \\
\hline 0.252 & 0.165 & 32.9977 & 32.99779 & 0.000288 \\
\hline 0.252 & 0.15 & 29.99807 & 29.99795 & -0.00039 \\
\hline
\end{tabular}


Decreasing the Breadth(b) and Depth(d) of the beam by $5 \%$

By using the polynomial regression method the natural frequencies of cantilever beam for decreasing the breadth(b) and depth(d) by $5 \%$ are as follows:

$f n=\alpha_{1}+\alpha_{2} b+\alpha_{3} d+\alpha_{4} b^{2}+\alpha_{5} d^{2}+\alpha_{6} b d$

$\alpha_{1}=-4.5607194547678632 \mathrm{E}-05 \quad \alpha_{2}=6.3630095325351022 \mathrm{E}-01$

$\alpha_{3}=1.9946185738721016 \mathrm{E}+02 \quad \alpha_{4}=2.3860827295949868 \mathrm{E}+01$

$\alpha_{5}=1.8618245239608640 \mathrm{E}+01 \quad \alpha_{6}=-4.2252242462718542 \mathrm{E}+01$

Table: Decreasing the Breadth(b) and Depth(d) of the beam by $5 \%$

\begin{tabular}{|c|l|r|r|c|}
\hline Breadth(b) & Depth(d) & fn (FEM) & fn (Regression) & \%Error \\
\hline 0.252 & 0.3 & 59.99529 & 59.99549 & 0.00033 \\
\hline 0.2394 & 0.285 & 56.99658 & 56.99587 & -0.00124 \\
\hline 0.2268 & 0.27 & 53.99553 & 53.99624 & 0.001318 \\
\hline 0.2142 & 0.255 & 50.99668 & 50.99659 & -0.00018 \\
\hline 0.2016 & 0.24 & 47.99703 & 47.99682 & -0.00021 \\
\hline 0.189 & 0.225 & 44.99724 & 44.99724 & $-1.1 \mathrm{E}-05$ \\
\hline 0.1674 & 0.21 & 41.99731 & 41.99784 & 0.00126 \\
\hline 0.1548 & 0.195 & 38.99881 & 38.99783 & -0.00251 \\
\hline 0.1422 & 0.18 & 35.99753 & 35.9978 & 0.000757 \\
\hline 0.1296 & 0.165 & 32.99693 & 32.99776 & 0.002499 \\
\hline 0.117 & 0.15 & 29.99807 & 29.99769 & -0.00124 \\
\hline
\end{tabular}

\section{Results}

From this work the following results are drawn. Natural frequencies of the cantilever beam are obtained for dynamic analysis of the beam from conventional equations, ANSYS 10.0 software, FEM using MAT LAB and polynomial regression method by considering the various situations. The maximum and minimum errors are obtained when the results of regression method are compared with conventional equations, FEM and ANSYS.

Table: Results Comparison For Cantilever Beam

Table 6.1 Results Comparison For Cantilever Beam

\begin{tabular}{|c|c|c|c|c|c|c|c|}
\hline \multirow{2}{*}{\multicolumn{2}{|c|}{ Situations }} & \multicolumn{2}{|c|}{ Conventional } & \multicolumn{2}{|c|}{ ANSYS } & \multicolumn{2}{|c|}{ FEM } \\
\hline & & Maximum & Minimum & Maximum & Minimum & Maximum & Minimum \\
\hline 1.Inc & ng by $5 \%$ & -0.0496 & -0.05806 & 0.001599 & -0.00246 & 1.776069 & -0.36733 \\
\hline $\begin{array}{l}\text { I. } \\
\text { II. }\end{array}$ & $\begin{array}{l}\operatorname{Depth}(\mathrm{d}) \\
\text { Breadth(b) and } \\
\operatorname{Depth}(\mathrm{d})\end{array}$ & -0.0504 & -0.05136 & 0.193028 & -1.40498 & 0.006197 & $-8.6 \mathrm{E}-05$ \\
\hline 2. Dec & ing by $5 \%$ & -0.02103 & -0.03809 & 0.00936 & -0.01084 & 0.0015 & -0.00046 \\
\hline $\begin{array}{r}\text { I. } \\
\text { II. }\end{array}$ & $\begin{array}{l}\operatorname{Depth}(d) \\
\text { Breadth(b) and } \\
\operatorname{Depth}(d)\end{array}$ & 0.076693 & -0.09864 & 0.005564 & $-3.1060 \mathrm{E}-06$ & 0.002499 & $-1.1 \mathrm{E}-05$ \\
\hline
\end{tabular}

\section{Conclusions}

\section{Conclusions \& Future Scope}

The following are the conclusions drawn from the work

a. The Natural frequencies of the Cantilever beam by using conventional equations and ANSYS 10.0 software are exactly equal.

b. The results obtained from FEM are approximately nearer to conventional results. By considering more number of elements we get nearer values.

c. The Reanalysis was carried out using Polynomial Regression Method for the four situations considered in the case study.

\section{References}

[1]. Uri kirsch, Michaelbogomolni, Analytical modification of structural natural frequencies, Department of civil and environmental engineering ,Technion- Israel institute of technology, Haifa 32000, Israel (2006).

[2]. B.P.Wang, Improved Eigen solution Reanalysis procedures in structural dynamics, department of mechanical engineering, Los Angeles, California (1990)

[3]. P.B.Nair, Approximate static and dynamic reanalysis techniques for structural optimization, Modern practice in stress and vibration analysis, Gilchrist(ed.).

[4]. M.M.Segura and J.T.celigilete, A new dynamic reanalysis technique based on model synthesis" "Centro de studios e investigaciones tecnicas", deguipuzcoa(CEIT) apartado 1555, 20009 san Sebastian, spain (1994).

[5]. Nataša Trišović, Modification of the dynamics characteristics in the structural dynamic reanalysis,facta universitatis, series: mechanical engineering vol. 5, no 1, 2007, pp. 1 - 9 .

[6]. Natasa Trisovic, Tasko Maneski, Tomislav Trisovic, Ljubica Milovic, Modification of the Dynamics characteristics using a Reanalysis Procedures Technique - New ResultsVol. 16, No. 1, 2012, ISSN 2303-4009 (online), p.p. 219-222. 
[7]. Sertac Koksal, Mutlu D. Comert, H. Nevzat Ozguven, Reanalysis of Dynamic Structures UsingSuccessive Matrix Inversion Method.

[8]. M. Cheikh and A. Loredo, Static reanalysis of discrete elastic structures with reflexive inverse, Volume 26, Issue 9, September 2002, Pages 877-891(ELSEVIER).

[9]. Burcu Sayin and Ender Cigeroglu, A new structural modification method with additional degrees of freedom for dynamic analysis of large systems(ELSEVIER).

[10]. Ezedine Allaboudi, Tasko Maneski, Natasa Trisovic, Todor Ergic, Improving structure dynamic behaviour using a reanalysis procedures technique, Original scientific paper, ISSN 1330-3651 (Print), ISSN 1848-6339 (Online).

[11]. Zhong-Sheng Liu, Su-Huan Chen, Reanalysis of static response and its design sensitivity of locally modified structures,Communications in Applied Numerical Methods, Volume 8, Issue 11, pages 797-800, November 1992.

[12]. Z. Xie, W.S. Shepard Jr," Development of a single-layer finite element and a simplified finite element modelling approach for constrained layer damped structures", Finite Elements in Analysis and Design 45 (2009) pp530-537.

[13]. Natasa Trisovic, Eigenvalue Sensitivity Analysis in Structural Dynamics.

[14]. Tirupathi R. chandrupatla, Ashok D. Belegundu, Introduction to finite elements in engineering (India: Dorling Kindersely,2007).

[15]. T K Kundra, Structural dynamic modifications via models,SaÅdhanaÅ, Vol. 25, Part 3, June 2000, pp. $261 \pm 276$.

[16]. R.C. Mohanty, B.K. Nanda,damping of layered and jointedbeams with riveted joints.

[17]. Julio f. davalos, Pizhong qiao, Multiobjective material architecture optimization ofpultruded frp i-beams," composite structures (1996).

[18]. Uri kirsch, G.Tolendano,"Finite Elements in Analysis and Design "Department of Civil and Environmental engineering, Technion Israel institute of technology, Haifa 32000, Israel.

[19]. Bates, D.M. and Watts, D.G., Nonlinear regression analysis and its applications, Wiley and Sons, New York, 1988.

[20]. Wells, J.W., Analysis and interpretation of binding at equilibrium, in Receptor-Ligand Interactions: A PracticalApproach, E.C. Hulme, Ed., Oxford University Press, Oxford, 1992, 289-395.

[21]. Natasa Trisovic, Tasko Maneski, Zorana Golubovic, Stefan Segla,Elements of Dynamic ParametersModification and Sensitivity.

[22]. Y.Dere, E. D. Sotelino,Solution of transient nonlinear structural dynamics problems using the modified iterative groupimplicit algorithm, ICCST '02 Proceedings of the sixth conference on Computational structures technology, Pages 81-82, CivilComp press Edinburgh, UK, UK @2002.

[23]. Jasbir S. Arora, Optimization of Structural and Mechanical Systems.

[24]. Uri kirsch,"Finite Elements in Analysis and Design "Department of Civil and Environmental engineering, Teknion Israel institute of technology, Haifa 32000, Israel.

[25]. Uri kirsch , Michael Bogomolni, Nonlinear and dynamic structural analysis using combined approximations, computers \& structures 85, (2007).

[26]. M. Nad'a , Structural dynamic modification of vibrating systems, Applied and Computational Mechanics 1 (2007), $203-214$.

[27]. W.T. THOMSON, Theory of vibration with applications (London W1V 1FP : Prentice-Hall, 1972).

[28]. Motulsky, H.J., Analyzing Data with GraphPad Prism, GraphPad Software Inc.,San Diego, CA, 1999.

[29]. Ludbrook, J., Comparing methods of measurements, Clin. Exp. Pharmacol. Physiol.,24(2), (1997) $193-203$.

[30]. Tao Li, Jimin He, Local structural modification using mass and stiffness changes, Engineering structures,21(1999), $1028-1037$.

[31]. Eligilete, A new dynamic reanalysis technique based on modal synthesis, computers \& structures,vol. 56 (1995),523-527.

[32]. Johnson, M.L. and Faunt, L.M., Parameter estimation by least-squares methods, Methods Enzymol (1992), $210,1-37$.

[33]. W.H., Teukolsky, S.A., Vetterling, W.T., and Flannery, B.P., Numerical recipesin C. The art of scientific computing, Cambridge University Press, Cambridge, MA, 1992.

[34]. Gunst, R.F. and Mason, R.L., Regression analysis and its applications: A data oriented approach, marcel dekker, New York,1980.

[35]. Cornish-Bowden, A., Analysis of enzyme kinetic data, Oxford University Press,New York, 1995.

[36]. Johnson, M.L., Analysis of ligand-binding data with experimental uncertainties in independent variables, MethodsEnzymol (1992), $210,106-17$. 